\title{
Efecto de Abonos Orgánicos sobre las Poblaciones de Nematodos y la Producción de la Papa
}

\author{
Lucio Iriarte ${ }^{1}$, Javier Franco ${ }^{2}$ y Noel Ortuño ${ }^{2}$
}

\section{Resumen}

En la comunidad de Mishka Mayu (3400 msnm) de la provincia Carrasco (Cochabamba, Bolivia) se estudió el efecto de la incorporación de abono verde y estiércol de bovino sobre la multiplicación de Nacobbus aberrans, Globodera spp., el rendimiento de la variedad Waych'a (Solanum tuberosum ssp. andigena) y su impacto económico. El abono verde proveniente del cultivo de haba (Vicia faba) se aplicó en tres dosis (5, 10 y 15 t/ha) y en dos formas (surco y voleo), mientras que el estiércol se aplicó al surco en tres niveles $(0,5$ y $10 \mathrm{t} / \mathrm{ha}$ ) que se compararon con un testigo correspondiente a la siembra tradicional de los agricultores (10 t/ha).

La rotación del cultivo de haba redujo en un 30\% la población inicial de $N$. aberrans y Globodera spp., con una tasa de multiplicación de 0.7 para ambos nematodos. Los mejores rendimientos se obtuvieron con la aplicación de abono verde al voleo en niveles de 5 a 10 t/ha, porque en cantidades superiores se observó un efecto fitotóxico. La respuesta al estiércol fue de tipo lineal, con un incremento en el rendimiento de 0.8 toneladas por cada tonelada incorporada. Los mayores rendimientos $(26.6 \mathrm{t} / \mathrm{ha})$ se obtuvieron con las combinaciones de abono verde al voleo y estiércol en la proporción de 5-10 y con la aplicación al surco, (24.8 t/ha) de la combinación 55 que superaron al testigo (19.2 t/ha). Cuando los niveles de 5 y 10 t/ha de abono verde, aplicados al voleo o al surco se ajustaron a las cantidades equivalentes efectivas que se incorporaron al surco, éstas fueron de 2.2 a $4.3 \mathrm{t} / \mathrm{ha}$ y de 5 a $10 \mathrm{t} / \mathrm{ha}$, respectivamente. Estos volúmenes representan una incorporación de 0.15 a $0.30 \mathrm{~kg}$ por metro de surco a un distanciamiento de $0.7 \mathrm{~m}$ entre surcos. Asimismo, la combinación 5-5 de abono verde y estiércol presentó la menor tasa de multiplicación de $N$. aberrans y $G$. pallida y el mayor ingreso económico al agricultor, que con el sistema habapapa obtuvo USS 5.9 por cada dólar invertido.

Tesis para obtener el título de Ingeniero Agrónomo, UMASS, Cochabamba, Bolivia.

Departamento de Nematología, Programa de Investigación de la Papa (PROINPA), Casilla 4285, Cochabamba, Bolivia. 
Palabras claves:

Globodera spp., Nacobbus aberrans, abono verde, estiércol, rendimientos, ingreso económico.

Aceptado para publicación: febrero 1999

\title{
Effect of Organic Fertilizers on Nematode Populations and Potato Production
}

\begin{abstract}
Summary
The effect of green and cow manure incorporation on Nacobbus aberrans and Globodera spp. multiplication rate, potato yield and economic impact was studied under field conditions in the rural community of Mishka Mayu (3400 masl) in Cochabamba, Bolivia.

The canopy of a previous Vicia faba crop was used as green manure at three rates (5, 10 and $15 \mathrm{t} / \mathrm{ha}$ ) and two incorporation forms (row and broadcast) whereas cow manure was applied as a row treatment also at three different rates ( 0,5 and $10 \mathrm{t} / \mathrm{ha}$ ). All combinations were compared against a traditional farmer field (10 t/ha of cow manure). The previous $V$. faba crop decreased both initial nematode soil densities in 30\%. Regarding the potato crop development in response to forms and rates of green and cow manure application, the best yields were obtained with the combination of $5 \mathrm{t} / \mathrm{ha}$ of green manure as broadcast and 10 of cow manure (26.6 t/ha), followed by the combination 5-5 as row application (24.8 t/ha) which were superior to the check (19.2 t/ha). In general, rates of green manure higher than $10 \mathrm{t} /$ ha were phytotoxic but potato yields were increased by $0.8 \mathrm{t}$ for each ton of cow manure incorporated. However, when the green manure rates of 5 and 10 t/ha applied as row or broadcast, were adjusted to the effective amounts applied to each meter of row, the corresponding rates were of 2.2 and $4.3 \mathrm{t} / \mathrm{ha}$, respectively or 0.15 and $0.30 \mathrm{~kg} / \mathrm{m}$ of row. In the same way the combination of $5-5 \mathrm{t} / \mathrm{ha}$ gave the lower rate of multiplication for both nematodes and the higher economic benefit for the system $V$. faba - potato, since farmers can obtain US\$ 5.9 per each invested dollar.
\end{abstract}


Key words:

Globodera spp., Nacobbus aberrans, green manure, cow manure, yields, economic benefit.

\section{Introducción}

Los principales nematodos que atacan al cultivo de la papa en la zona andina de Bolivia son Nacobbus aberrans, conocido como el "rosario de la papa" y Globodera spp. o "nematodo quiste de la papa". Estos nematodos individualmente 0 en interacción causan pérdidas del 30 al 70 $\%$ en los rendimientos del cultivo de papa en Bolivia (Franco, 1994).

En la actualidad, el uso indiscriminado de productos químicos con ingredientes activos de alta toxicidad y de prolongado efecto residual es la causa del desequilibrio ecológico y de la alteración de la biología del suelo (Bird, 1987). Este hecho, ha motivado la intensificación del empleo de diversos métodos de control biológico para disminuir las poblaciones de nematodos y entre éstos está la práctica de abonamiento en verde, que es la incorporación de plantas jóvenes al suelo en el lugar donde se cultivaron y el empleo de materia orgánica en forma de estiércoles animales.

Numerosos estudios han demostrado que la incorporación de materia orgánica constituye una alternativa importante en la protección de cultivos. El daño causado por nematodos no es tan severo en suelos enriquecidos con materia orgánica como en suelos con bajo contenido de ésta. La aplicación de materia orgánica en forma de compost mejora las propiedades físicas del suelo y promueve un mejor desarrollo de la planta, proporcionando mayores rendimientos a pesar de la presencia de los nematodos.

Van der Laan (1956) indica que la incorporación de materia orgánica retarda el desarrollo de juveniles de Globodera spp. dentro de las raíces y simultáneamente disminuye su densidad poblacional. Además Duddington (1957) indica que la materia orgánica estimula cierto grado de tolerancia por parte de las plantas y favorece la aparición e incremento de enemigos naturales de los nematodos, intensificando la acción de hongos predatores.

La incorporación de abonos verdes, constituye otra de las formas de adición de materia orgánica a los suelos y también se ha observado que incrementa los rendimientos y disminuye la infestación de los 
nematodos en el suelo. La incorporación de lupinos, trébol dulce y mostaza (Ellenby, 1945; Koltermann, 1943) dio resultados favorables. Koltermann (1943) encontró que la incorporación en verde de mostaza incrementó los rendimientos en 65\% y disminuyó la invasión del nematodo quiste de la papa en $25 \%$. Sin embargo, no siempre se observa la disminución en el número de quistes. Es así que Schmidt (1952) obtuvo luego de incorporar lupinos y mostaza mayores cosechas pero también mayor número de quistes. Mas aún, la incorporación de trébol como abono verde en el cultivo de la papa puede incrementar la densidad de Pratylenchus penetrans a niveles que causan daños considerables.

En estudios con Heterodera schachtii, se observó que la descomposición de trébol dulce (Melilotus sp.) incorporado al suelo, elevó la temperatura y liberó diversos gases que provocaron la destrucción de 5 a 22\% de las población de nematodos del suelo, inclusive dentro de los quistes (Thorne, 1926). De otro lado se observó que la incorporación de alfalfa como abono verde, es mucho más eficiente para controlar Meloidogyne spp. (Sharma et al., 1982).

En base a estos antecedentes se investigó la eficiencia de la incorporación de haba (Vicia faba L.) en verde combinado con dosis de estiércol de bovino sobre las poblaciones de $N$. aberrans y Globodera spp., y la producción de papa y su impacto económico.

\section{Materiales y Métodos}

El presente estudio se realizó en campos naturalmente infestados con Globodera spp. y N. aberrans y con disponibilidad de agua de riego de la comunidad de Mishka Mayu (3100 a $3400 \mathrm{msnm}$ ) perteneciente a la provincia Carrasco del departamento de Cochabamba (Bolivia), cuyo muestreo se realizó previamente.

En primer lugar, se efectuó la siembra del cultivo de haba (25 de agosto) y después de la primera cosecha de vainas (4 y 5 de febrero), se incorporó el follaje en dosis de 5, 10 y 15 t/ha, bajo dos formas de aplicación o distribución al suelo (voleo y surco).

Simultáneamente se establecieron los tratamientos con estiércol de bovino $(0,5$ y 10 t/ha) más un testigo adicional correspondiente a la siembra tradicional del agricultor con 10 t/ha de estiércol. La fertilización base utilizada para todos los tratamientos, incluido el testigo, fue 80-1200 . Una vez establecidos los tratamientos (6 de febrero), se sembró el cultivo de papa con el cv. Waych'a (Solanum tuberosum ssp. andigena), susceptible a Globodera spp. y N. aberrans. 
El efecto de los diversos tratamientos se determinó por las variables agrofisiológicas y nematológicas que se indican para cada cultivo.

Cultivo de haba. Se determinó el rendimiento en materia verde, vaina verde y la tasa de multiplicación $(\mathrm{TM}=\mathrm{Pf} / \mathrm{Pi})$ de $N$. aberrans y Globodera spp., al inicio y luego de la incorporación del cultivo de haba, respectivamente.

Cultivo de papa. Se empleó un diseño estadístico de parcelas divididas $(2 \times 3 \times 3)$ con tres repeticiones. Las parcelas representaban las formas de aplicación (voleo y surco) del abono verde (AV), las sub parcelas por sus niveles y las sub-sub parcelas estaban representadas por los niveles de estiércol. Los parámetros evaluados fueron el porcentaje de emergencia, porcentaje de cobertura, peso de la raíz por planta, número de tubérculos por planta, rendimiento total y peso de tubérculos según su tamaño o categoría. Asimismo, se determinó la tasa de multiplicación de ambos nematodos por muestreos de suelo efectuados a la siembra y cosecha del cultivo de papa. El número de nódulos en la raíz ocasionados por $N$. aberrans se determinó a la floración de las plantas de papa.

La identificación de la especie de Globodera spp. presente en los campos experimentales, se efectuó por las características morfológicas de 25 quistes extraídos de muestras de suelo y las de segundos estados juveniles provenientes de los mismos quistes (González y Franco, 1993).

Las poblaciones de $N$. aberrans y Globodera spp. extraídas de las muestras de suelo en diversas oportunidades se determinaron por el procesamiento de 100 gramos de suelo por los métodos de tamizado más centrifugación y el embudo de Fenwick modificado, respectivamente (Montecinos y Franco, 1.993).

Finalmente para determinar el tratamiento más favorable para el agricultor, se efectuó el análisis económico del sistema haba-papa por el cálculo de la Tasa de Retorno Marginal (CIMMYT, 1988). 


\section{Resultados y Discusión}

\section{Cultivo de haba}

\section{Evaluaciones agrofisiológicas:}

El rendimiento de materia verde, luego de la primera cosecha de haba de 71 t/ha y el rendimiento en vaina verde, presentó una media de 10.2 t/ha, superior a la media nacional de 7 t/ha (Vera, 1992). El estado ideal de desarrollo de la planta de haba para su incorporación como abono verde (AV), es inmediatamente después de la primera cosecha, debido a que presenta el mayor peso de materia verde y el mayor peso de materia seca (MS) que oscila de 77 a $89 \%$ en el follaje y 11 a $20 \%$ en la raíz, comparado con los demás estados de desarrollo.

\section{Evaluaciones nematológicas:}

Primeramente se identificó la población de Globodera spp. presente en el campo y se determinó el efecto del cultivo de haba sobre la población de nematodos existentes en el suelo.

a) Identificación de Globodera spp.: En los quistes del nematodo se determina el número de líneas entre la fenestra vulvar y el ano, y la forma ovalada de la fenestra, identificándose a la especie G. pallida. La proyección anterior de los nódulos del estilete de los segundos estados juveniles (J2) confirmó esta identificación.

b) Efecto sobre la población de $N$. aberrans y G. pallida: La incorporación de haba mostró una disminución del $30 \%$ de la población inicial de $N$. aberrans y G. pallida, con una Tasa de Multiplicación $(\mathrm{TM}=\mathrm{Pf} / \mathrm{Pi})$ de 0.7 , indicando que no existió reproducción de los nematodos.

\section{Análisis económico:}

El ingreso por la venta de haba en vaina verde, fue de US\$2,125 y como valor de fertilizante se tuvo un ingreso de US\$992.85, haciendo un total de US\$3,117.85 por ha. 


\section{Cultivo de papa}

\section{Evaluaciones agrofisiológicas:}

Se determinó el desarrollo de las plantas de papa y su rendimiento total como respuesta a los diversos tratamientos de abono verde y estiércol de bovino.

a) Desarrollo de la planta: La incorporación de hasta 10 t/ha de AV al voleo y al surco en combinación con 5 y 10 t/ha de estiércol favoreció al desarrollo de la planta (emergencia, cobertura y altura de planta). El nivel de 15 t/ha de AV al voleo o al surco afectó en forma negativa el desarrollo de la planta. Los niveles puros de AV al voleo y al surco, no suplieron al estiércol.

b) Rendimiento: La producción de tubérculos se analizó en forma detallada para establecer las ventajas agroecológicas y económicas del sistema bajo estudio, porque de éste dependerá la alimentación y el ingreso económico del agricultor.

Efectuado el análisis de varianza se encontró únicamente diferencias significativas para los efectos principales (forma de aplicación, niveles de abono verde y para el factor estiércol) indicando que cada factor actuó en forma independiente y por lo cual, los análisis de comparación se efectuaron también en forma independiente.

Para el factor estiércol se observó una tendencia lineal positiva en el rendimiento al incremento de estiércol. Por cada tonelada de estiércol aplicada a la siembra existió una respuesta inmediata de la planta en el rendimiento de $0.8 \mathrm{t} / \mathrm{ha}$. Esta respuesta es corroborada por la alta correlación existente $(r=0.95)$ entre los datos observados y los calculados (Figura 1).

Según la forma de aplicación del AV, la mayor producción de tubérculos se obtuvo con la aplicación al voleo (20.3 t/ha como promedio), la menor producción con la aplicación al surco (18.1 t/ha como promedio). 


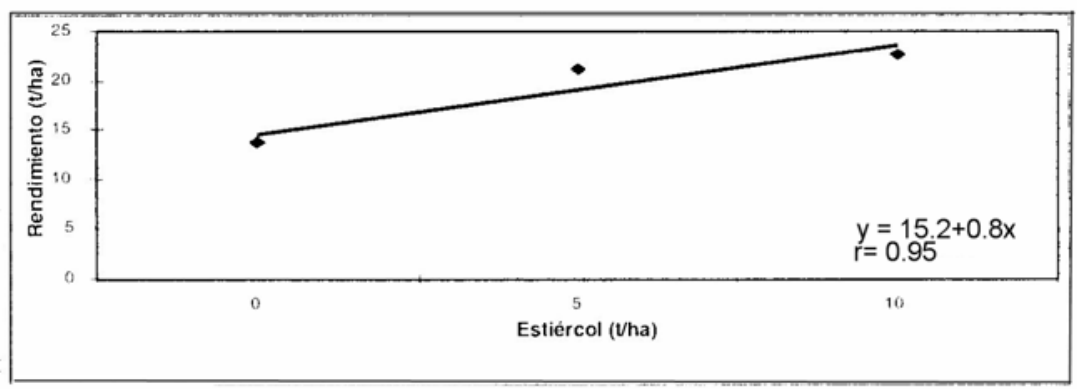

Figura 1. Efecto de los niveles de estiércol sobre el rendimiento total de papa.

Cuando se incrementó el $\mathrm{AV}$, se observó una disminución en el rendimiento total de $0.27 \mathrm{t} / \mathrm{ha}$, por cada tonelada de AV incorporada (Figura 2). Esta tendencia lineal descendente mostró una alta correlación negativa entre los datos observados y calculados. La superioridad observada del nivel de $10 \mathrm{t}$ de abono verde, probablemente se deba a que éste actuó como mejorador de las propiedades físicas (estructura) y no así como mejorador de las propiedades químicas del suelo. Por este efecto, también se puede asumir que los niveles bajos de AV (5 y 10 t) en los que se observó un incremento de 19.99 a 20.33 t/ha, son los niveles óptimos de incorporación al suelo y no así el nivel de 15 t (17.31 t/ha) que muestra un posible efecto fitotóxico y que su efecto residual se observaría en el siguiente cultivo, es decir, cuando éste se descomponga totalmente.

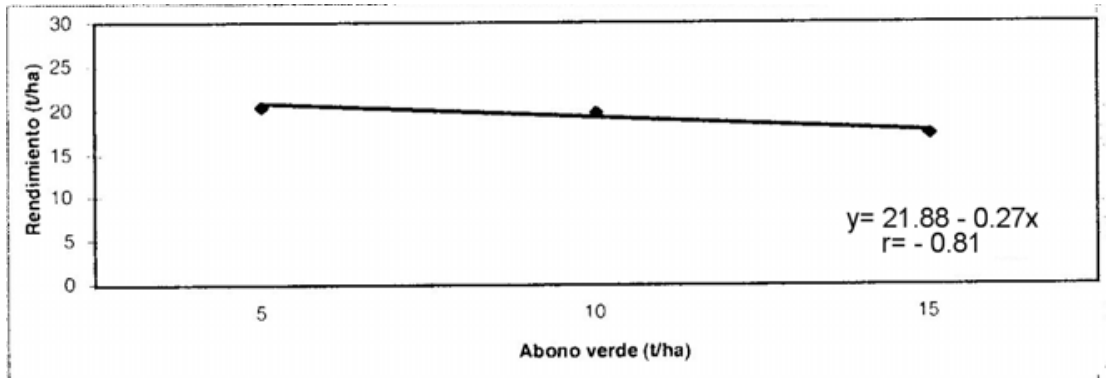

Figura 2. Efecto de diferentes niveles de abono verde sobre el rendimiento del cultivo de papa, cv. Waych'a 
Sin embargo, aún cuando cada factor estudiado actuó en forma independiente y no se determinaron interacciones significativas entre ellos, se tiene que los mayores rendimientos de papa se obtuvieron con la aplicación de AV al voleo (5 y 10 t/ha) en combinación con niveles similares de estiércol, cuyos rendimientos fueron 23.5 t/ha para la combinación 5-5, 26.6 t/ha para 5-10 y 25.5 t/ha para 10-10 y los tratamientos al surco con rendimientos de 23.3 t/ha con la misma combinación de 10-10 y de 24.8 t/ha para 5-5 que fueron superiores al testigo que tuvo un rendimiento de $19.2 \mathrm{t} / \mathrm{ha}$.

Por otro lado, cuando estos niveles de aplicación de AV (5, 10, 15 t/ha) se ajustan a la cantidad efectiva disponible de kilos por metro de surco a un distanciamiento de $0.70 \mathrm{~m}$ de acuerdo al sistema de aplicación (voleo y surco), se obtienen cantidades equivalentes diferentes de AV incorporado.

Al relacionarse estas cantidades de incorporación de AV por metro de surco con los rendimientos obtenidos (Figura 3), se observa que la cantidad óptima de aplicación de abono verde para obtener los mayores rendimientos y para evitar efectos de fitotoxicidad, sería de 5.0 a 10.0 t/ha en aplicación al voleo y de 2.2 a 4.3 t/ha en aplicación al surco, que corresponden a los valores de 0.150 y 0.300 kilos de abono verde de haba por metro de surco, antes de la siembra del cultivo de papa. De sobrepasarse estas cantidades se observa una disminución en el rendimiento tal como lo muestra la ecuación polinómica calculada para los valores equivalentes de AV aplicados en kilogramos por metro de surco.

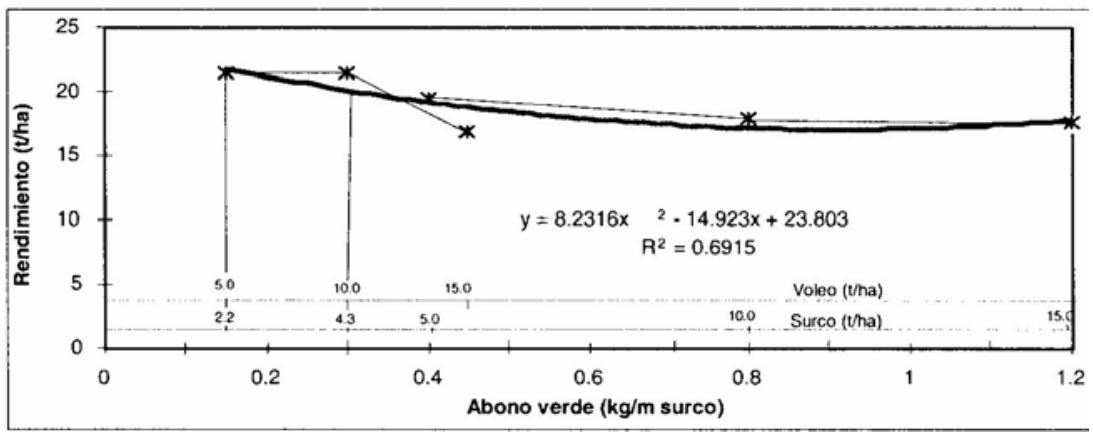

Figura 3. Rendimiento de papa, cv. Waych'a (tha), de acuerdo a cantidades estimadas de abono verde $(\mathrm{kg})$ por metro de surco en las aplicaciones al voleo y al surco (tha). 


\section{Evaluaciones nematológicas:}

El efecto de los diversos tratamientos sobre las poblaciones de $N$. aberrans y Globodera spp., se analizaron de acuerdo a los parámetros nematológicos que se presentan a continuación.

a) Número de nódulos de $N$. aberrans : Aún cuando ocurrió la formación de nódulos en las plantas de todos los tratamientos (incluido al testigo), se observaron diferencias para la forma de distribución del abono verde. La distribución al voleo presentó el mayor número de nódulos (2.95 datos transformados), comparado con lo aplicado al surco (2.7). Esta diferencia en el número de nódulos se debería a que el AV aplicado al surco protegió mejor la raíz como resultado de su mayor concentración y que los nódulos observados se deberían a que la raíz salió fuera de la protección o acción del AV.

b) Tasa de Multiplicación (TM): La Tasa de Multiplicación de N. aberrans fue similar en todos los tratamientos (incluido el testigo). No obstante existió diferencia en la forma de aplicación del abono verde, ya que la aplicación al voleo presentó 1.25 y al surco 1.09 (datos transformados). Esta diferencia se debería también a que la aplicación al surco protegió mejor las raíces del ataque del nematodo probablemente por la liberación del $\mathrm{CO}_{2}, \mathrm{NH}_{3}$ y aumento de temperatura como resultado del proceso de descomposición del abono verde (Bello et al., 1997).

c) Para la distribución del AV al voleo, la TM para G. pallida, mostró una tendencia descendente a medida que se incrementó el estiércol (Figura 4). En cambio, con la aplicación al surco, aún cuando la TM fue también baja, se mostró ligeramente ascendente debido probablemente a que las raíces por su mayor desarrollo con la dosis alta de estiércol (10 t/ha), llegaron a salir de la protección del AV y fueron invadidas por el nematodo. Por el contrario, la menor tasa de multiplicación con el AV aplicado al surco pero con dosis bajas de estiércol, se debería al menor desarrollo radical ocurrido a la mayor concentración del AV debido a que la mayor cantidad de AV, generó productos tóxicos durante el proceso de descomposición, que se llegaron a concretar por unidad de área en el mismo lugar donde se colocó el AV y afectó la formación de nuevos quistes y su viabilidad total. 


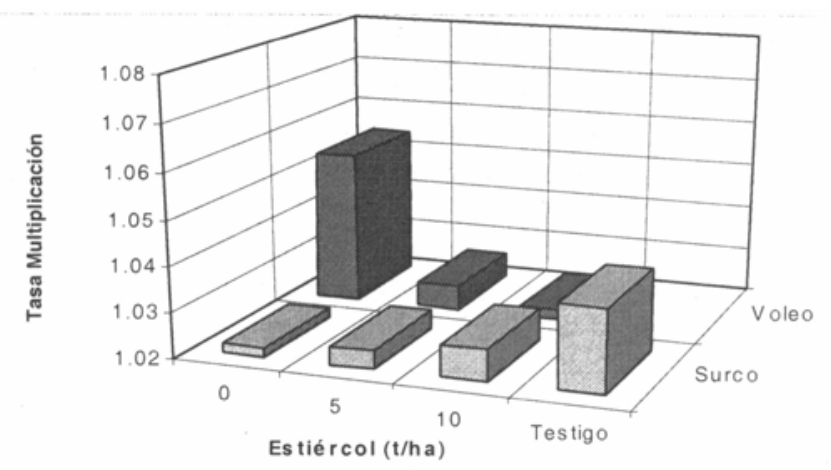

Figura 4. Efecto de la aplicación del abono verde (voleo y surco) y tres niveles de estiércol sobre la Tasa de Multiplicación de Globodera pallida (datos transformados).

\section{Sistema Haba-Papa}

\section{Efecto sobre la producción agrícola:}

Los resultados obtenidos con ambos cultivos en este sistema propuesto son una alternativa para el mejoramiento de los suelos, como también dentro de un sistema de rotación para disminuir las poblaciones de nematodos y favorecer posteriormente el rendimiento del cultivo de papa.

\section{Efecto sobre la población de N. aberrans y G. pallida:}

Las poblaciones de nematodos con esta rotación y con la incorporación de materia verde disminuyeron su poder infectivo y el nivel de infestación del suelo.

Nacobbus aberrans disminuyó su población y su poder infectivo durante el desarrollo del cultivo de papa, debido a la descomposición del abono verde, liberando gases tóxicos (NH3), como también otras sustancias que inhiben la eclosión de los huevos (Bello et al., 1997).

En cambio, G. pallida fue afectado en sus diferentes estados juveniles, por la incorporación de AV y su posterior descomposición. La fertilidad de las hembras disminuyó, lo cual se manifestó en el menor diámetro de los quistes nuevos y por ende, dentro de cada quiste hubo menor número de huevos viables, disminuyendo la viabilidad de cada quiste entre 40 a 60\% y el nivel de infestación del suelo. 


\section{Análisis económico:}

El análisis económico consideró la superficie del cultivo de haba para producir 5,10 y 15 t/ha de materia verde (AV), el ingreso por la venta de haba (vaina verde) y la producción de papa para consumo. En base a los resultados obtenidos (Figura 5) se desprende que los tratamientos no dominados fueron las combinaciones de estiércol + abono verde de 0-5 al voleo y de 5-5 t/ha al voleo y al surco y el testigo 10-0 t/ha (tratamientos $1,4,13$ y $\mathrm{T}$, respectivamente).

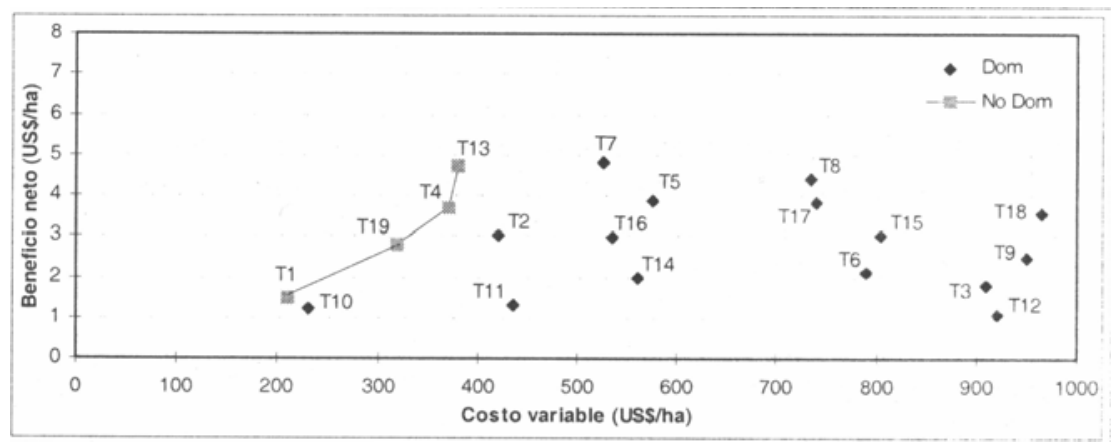

Figura 5. Discriminación de los diferentes tratamientos en la rotación de cultivos haba-papa.

El cálculo de la tasa de retorno marginal (TRM) en la Tabla 1, permite apreciar que el tratamiento 5-5 t/ha distribuido al surco (13) tiene la tasa de retorno marginal más elevada (5958\%), seguido del tratamiento 5-5 t/ha, distribuido al voleo (4) con $1212 \%$. Esto significa que económicamente la combinación 5-5 (5 t/ha AV de haba distribuido al surco + 5 t/ha estiércol), es la alternativa de producción en una rotación de cultivos de haba-papa en campos naturalmente infestados con nematodos, donde el agricultor obtendrá US\$ 5.9 por cada dólar invertido. 
Tabla 1. Tratamientos no dominados y estimación respectiva de los costos variables y tasa de retorno marginal (TRM).

\begin{tabular}{|c|c|c|c|c|c|}
\hline $\begin{array}{c}\text { Tratamiento } \\
\text { (Est. + AV) }\end{array}$ & $\overline{\mathrm{BN}(1)}$ & $\begin{array}{l}\text { Total } \\
\text { CV(2) }\end{array}$ & BNM (3) & CVM (4) & TRM (\%) \\
\hline 1 (0-5 voleo) & 2383.58 & 209.59 & & & \\
\hline $\mathrm{T}(10-0)$ & 3546.47 & 313.60 & 1162.89 & 104.09 & 1117.00 \\
\hline 4 (5-5 voleo) & 4185.38 & 366.31 & 638.91 & 52.7 & 1212.00 \\
\hline 13 (5-5 surco) & 5027.38 & 380.44 & 842.00 & 14.13 & 5958.00 \\
\hline
\end{tabular}

(1) Beneficio neto

(2) Costo variable

(3) Beneficio neto marginal

(4) Costo variable marginal

Es así que en base a los resultados obtenidos se tiene en primer lugar que la rotación con el cultivo de haba, disminuyó la población de Globodera pallida y $N$. aberrans, por ser éste un cultivo no hospedante de ambos nematodos.

Asimismo, la incorporación de 5 ó 10 t/ha de abono verde al surco o al voleo, respectivamente, al interaccionar con el estiércol favorecen el desarrollo de la planta, disminuyen la Tasa de multiplicación y el número de nódulos de $N$. aberrans y la Tasa de multiplicación de $G$. pallida. Niveles superiores de AV aún cuando reducen la población de nematodos, afectan negativamente el desarrollo de la planta, porque resultan en la disminución de los rendimientos de papa en 0.27 t/ha por cada tonelada adicional.

Por otro lado, se tiene que el estiércol es el abono orgánico más importante para el desarrollo de la planta y la producción de papa a nivel comercial, confirmando su rol de regulador de las propiedades físicas y biológicas del suelo ( $\mathrm{pH}$, estructura, textura). Cada tonelada incorporada produce un incremento de 0.8 t/ha en los rendimientos del cultivo de papa e interacciona favorablemente con aplicaciones de 5 y 10 t/ha de abono verde. Los mayores rendimientos de papa se obtienen con la aplicación de abono verde al voleo (10 t/ha) o al surco ( 5 t/ha) en combinación con los niveles de 5 y 10 t/ha de estiércol que fueron superiores al testigo (10 t/ha estiércol).

Por las ventajas obtenidas con el sistema de rotación o secuencia de cultivos, más la incorporación de materia verde del haba para disminuir las poblaciones de $N$. aberrans y Globodera spp., mejorar el suelo e incrementar los rendimientos del cultivo de papa en zonas con disponibilidad de riego se establece que la combinación 5-5 (estiércol-AV t/ha), que corresponde a $0.150 \mathrm{~kg}$ de abono verde por metro de surco, presentó la menor Tasa de multiplicación de ambos nematodos, 
el mejor rendimiento y el mayor ingreso económico para el agricultor. La mayor tasa de retorno marginal se obtiene por la venta de haba en vaina verde y papa para consumo, donde por cada dólar invertido se obtienen 5.9 dólares. Sin embargo, este nivel de 5 t/ha de abono verde se puede reducir a niveles de 2.2 a 4.3 t/ha, que representa una incorporación de 0.150 a 0.300 kilogramos por metro de surco y por lo tanto un menor volumen de abono verde. Para alcanzar estas cantidades por metro de surco y obtener rendimientos similares en aplicaciones al voleo se requerirían de 5 a 10 t/ha de abono verde, que en ambos casos se deben combinar con 5 t/ha de estiércol bovino.

\section{Referencias Bibliográficas}

1. Bello, A.; Gonzáles, J.A.; Tello, J.C. 1997. La biofumigación como alternativa a la desinfección del suelo. Horticultura Internacional 17, 41-43.

2. Bird, G. W. 1987. Role of Nematology in Integrated Pest Management Programs. En: Vistas on Nematology. J. A. Veech and D. W. Dickson (eds.). p. 114-121

3. Centro Internacional de Mejoramiento de Maíz y Trigo. 1988. La formulación de recomendaciones a partir de datos agronómicos: Manual metodológico de evaluación económica. México D. F., México.

4. Duddington, C. L. 1957. The Friendly Fungi. Faber and Faber. London:MacMillan, New York. 188 p.

5. Ellenby, C. 1945. The influence of crucifers and mustard oil on the emergence of larvae of the potato-root eelworm, Heterodera rostochiensis Wollenweber. Ann. Appl. Biol. 32: 67-70.

6. Franco, J. 1994. Problemas de nematodos en la producción de papa en climas templados en la región andina. Nematrópica 24 (2): 179-195.

7. González, A.; Franco, J. 1993. Manual de técnicas y métodos para estudios del nematodo quiste de la papa Globodera spp. Centro Internacional de la Papa (CIP). Lima, Perú, Programa de Investigación de la Papa (PROINPA). Cochabamba, Bolivia. 87 p.

8. Koltermann, D. 1943. Die Bedeutung der Kartoffelmudigkeit fur die Kartoffelwirtschaften. Mitt. Landwirtsch 58: 129-130. 
9. Mankau, R. 1968. Reduction of root-knot disease with organic amendments under semifield conditions. Plant Disease Rep. 52:315-319.

10. Montecinos, R.; Franco, J. 1993. Diagnóstico de los principales nematodos del cultivo de la papa. Manual Técnico No. 1/93. PROINPA. Cochabamba, Bolivia. 26 p.

11. Russell, W. 1968. Condiciones del suelo y crecimiento de las plantas. Madrid, España. 748 p.

12. Schmidt, J. 1952. Untersuchunger über den Kartoffelnematoden. In: Pflanzenschutztagung in Berlín, Deutsch. Bauernverlag, Berlin: 4953.

13. Sharma, R. D.; Pereira J.; Resck, D. V. S. 1982. Eficiencia de adubos verdes no controle de nematoides associados a soja nos Cerrados. Boletín de Pezquiza No. 13 EMBRAPA.

14. Thorne, G. 1926. Control of sugar-beet nematode by crop rotation. Washington, Department of Agriculture, USDA, Farmers Bulletin 1514.

15. Van der Laan, P. A. 1956. Onderzoekingen over schimmels, die parasiteren op de cyste-inhoud van het aardapelcystenaaltje (Heterodera rostochiensis Woll.). Tijdschr. PI. Ziekten, 62: 305321.

16. Vera, R. 1992. Efecto de la incorporación del haba en diferentes estadios de desarrollo. Tesis Ing. Agr. Universidad Mayor de San Simón. Cochabamba, Bolivia. 